\section{IN THE NEWS}

This year's Nobel prize as reported in La Vanguardia.

Three researchers who have revealed how the brain works win the Nobel

... Forty years ago ... the brain was a black box about which nothing was known ...; now, it is an organ whose workings are known in general terms and for which there are ... relatively efficacious drugs. This change in perspective is due, in large part, to the work of three pioneers who yesterday were awarded the Nobel Prize for Medicine: the Swede Arvid Carlsson, and the Americans Paul Greengard and Eric Kandel.

The work Arvid Carlsson has carried out for over forty years spans the whole spectrum of biomedical research, ranging from ... experiments with a substance few were willing to put money on dopamine - up to the design of drugs against Parkinson's, schizophrenia and depression ... [and] a series of studies that revealed how and where Parkinson's disease starts and how to treat it. Even nowadays, at his 77 years of age, "it's impressive how he continues to vibrate with research", Ernest Arenas, ... Catalan researcher at the Karolinska Institute, explained yesterday in a phone interview. "I met him first ... in 1998 at a meeting in Barcelona and was impressed by how, despite his age, he keeps on looking for better drugs against Parkinson's and still gets excited about his work".

When asked about what he will do with the [Nobel] prize money ...., Carlsson replied yesterday: "I first have to talk to my wife, she is the one who makes these decisions"...

Translated by Juan Carlos López

\title{
Vive la (subtle) différence!
}

The idea that genotypic differences among inbred strains of mice might explain the variability of their phenotypes may sound like a tautology.

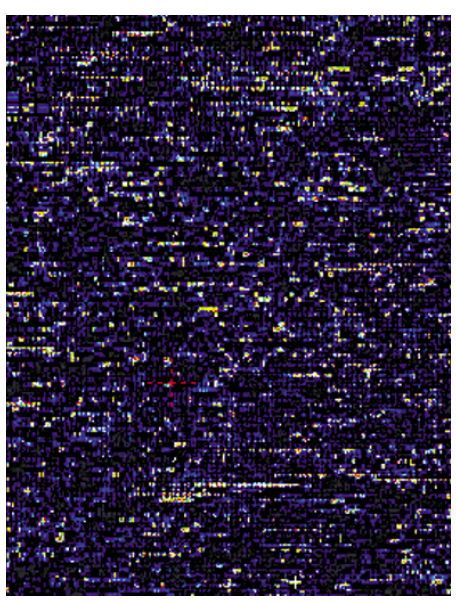

DNA array courtesy of Todd Carter, The Salk Institute, La Jolla, California, USA.
The fact is, however, that some of the brain phenotypes found in early studies of genetically modified animals were difficult to interpret because of differences in the genetic background of non-isogenic littermates. Therefore, it has become important to identify the genes that account for the phenotypic variability among strains and the use of DNA arrays has become a powerful tool to address this issue. Now, as Sandberg et al. report in the 26 September issue of PNAS, the differences in gene expression may be subtler than we think.

The authors used DNA arrays with more than 10,000 genes and searched for differences between two inbred mouse strains - 129SvEv and C57BL/6 - in the whole brain and across several brain regions. They found that only about $0.33 \%$ of the genes screened showed differential brain expression between the two strains. Moreover, a search for genes differentially expressed in at least one brain region across the two strains increased the fraction to a mere $1 \%$. It is noteworthy that the relatively low number of genes showing differential expression was not exclusive of the comparison between strains. Sandberg et al. used the same strategy to identify region-specific genes within the same strain and observed that only $0.54 \%$ of the genes screened showed clear differences.

Some of the genes identified in this screening correspond to previously known differences between strains or among regions, an observation that underscores the reliability of the approach. Some others could indeed help to explain a few of the differences in brain phenotypes documented between the two strains, such as their differential susceptibility to neurotoxic insult. A third group of genes consists of

NEUROACTIVE DRUGS

\section{Highs and lows of hippocampal inhibition}

Although not many would be prepared to admit to any first-hand knowledge, the psychoactive effects of marijuana are among those best known to the general public. Memory impairment is one of those effects (or so they tell me). What influence do cannabinoids exert on structures involved in memory, such as the hippocampus? Hajos et al. have approached this question by studying the cellular distribution of the cannabinoid receptor $\mathrm{CB} 1$ within the hippocampal formation, and by testing whether cannabinoid agonists affect GABA-mediated inhibition.

Using an antibody against the C-terminus of $\mathrm{CB} 1$, the authors found that the receptor was present only in a sub-population of inhibitory neurons, which also contained the peptide cholecystokinin. As CB1 was localized to the presynaptic terminals, Hajos et al. asked whether GABA-mediated transmission would be modulated upon the application of cannabinoid agonists. Indeed, the amplitude of evoked, calcium-dependent inhibitory postsynaptic potentials decreased in the presence of the CB1 agonists WIN55,221-2 and CP55,940. The drugs did seem to act presynaptically, as their application was associated with an increase in the number of failures of transmission elicited by minimal stimulation. In addition, CB1 agonists reduced the amplitude of

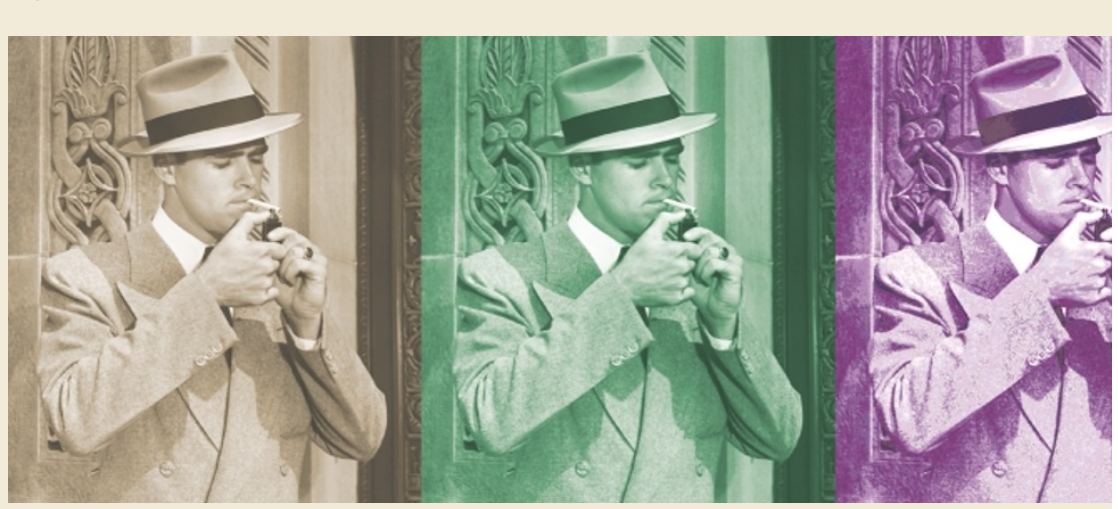

pharmacologically induced network oscillations that resemble a subset of those measured in vivo.

The authors propose that the action of CB1 agonists could result from the inhibition of voltage-gated calcium channels. Similarly, they suggest that the effect of cannabinoids on the network oscillations could be related to the effect of marijuana on memory. It is interesting to note that, as not every inhibitory neuron posseses $\mathrm{CB} 1$ receptors, it is possible 